\title{
Health-related Quality of Life and Toxicity After Single-fraction High-dose-rate Brachytherapy With External Beam Radiotherapy for Localized and Locally Advanced Prostate Cancer
}

\author{
TOMOYUKI MAKINO ${ }^{1}$, KAZUFUMI NAKASHIMA ${ }^{1}$, MASASHI IIJIMA ${ }^{1}$, \\ SHOUHEI KAWAGUCHI ${ }^{1}$, TAKAHIRO NOHARA ${ }^{1}$, KAZUYOSHI SHIGEHARA ${ }^{1}$, \\ KOUJI IZUMI ${ }^{1}$, YOSHIFUMI KADONO ${ }^{1}$, TOMOYASU KUMANO ${ }^{2}$ and ATSUSHI MIZOKAMI ${ }^{1}$ \\ ${ }^{1}$ Department of Integrative Cancer Therapy and Urology, \\ Kanazawa University Graduate School of Medical Science, Kanazawa, Japan; \\ ${ }^{2}$ Department of Radiotherapy, Kanazawa University Graduate School of Medical Science, Kanazawa, Japan
}

\begin{abstract}
Background/Aim: To evaluate the treatment outcomes, toxicity and health-related quality of life (HRQOL) in prostate cancer $(\mathrm{PCa})$ patients who underwent singlefraction high-dose-rate brachytherapy (single-fraction HDR$B T)$ with external beam radiotherapy (EBRT). Materials and Methods: From April 2014 to October 2017, treatment outcomes and toxicity of 85 patients who underwent singlefraction HDR-BT of 13 Gy, followed by 46 Gy EBRT in 23 fractions, were examined. HRQOL of 53 patients was evaluated using the Expanded Prostate Cancer Index Composite (EPIC), International Prostate Symptom Score (IPSS)/QOL index, International Index of Erectile Function 5 (IIEF-5), and 36-Item Short Form Survey (SF-36) scores through one year. Results: The median follow-up period was 28.8 months. Only three patients had biochemical recurrence. Toxicities included less than grade 3 lower urinary tract symptoms and grade 1 diarrhea. Urethral stricture, a problem related to late toxicity in conventional HDR-BT, was not observed. The urinary and bowel functions in EPIC scores significantly worsened until three or six months after treatment, respectively. Conclusion: Single-fraction HDR-BT with EBRT showed promising biochemical control, tolerant toxicities, and preservation of HRQOL, and can be efficiently performed in a shorter time than conventional HDR-BT.
\end{abstract}

Correspondence to: Atsushi Mizokami, MD, Ph.D., Department of Integrative Cancer Therapy and Urology, Kanazawa University Graduate School of Medical Science, 13-1 Takaramachi, Kanazawa, Ishikawa 920-8640, Japan. Tel: +81 762652393, Fax: +81 762344263,e-mail: mizokami@staff.kanazawa-u.ac.jp

Key Words: Prostate cancer, high-dose-rate brachytherapy, singlefraction, quality of life, toxicity.
With advances in radiation therapy techniques, there are currently several radiation therapy options for localized prostate cancer (PCa). High-dose-rate brachytherapy (HDRBT) is an effective treatment modality that can be used either alone or in combination with external beam radiotherapy (EBRT) for patients with localized PCa (1-5). The combined use of HDR-BT and EBRT allows considerable dose escalation while decreasing the dose administered to organs at risk, thereby improving PCa treatment outcomes. However, the conventional fractionated irradiation of HDR-BT modality has certain limitations, because it requires the patient to rest on the bed during the treatment period to avoid the accidental removal of the catheter from the perineum, and this may be associated with an increased risk of deep vein thrombosis. To date, it has been reported that the administration of a singlefraction HDR-BT protocol resulted in a high disease control rate and low toxicity (6-13), but there are few institutions in Japan performing a single-fraction HDR-BT protocol, and there is no report from this country. We started a HDR-BT modality from February 1999 in our hospital and previously reported the usefulness of HDR-BT with EBRT (5), but we have changed the single-fraction HDR-BT protocol since April 2014 to relieve the burden of patients.

In this study, we intended to evaluate treatment outcomes, safety, and impact on health-related quality of life (HRQOL) in patients with localized and locally advanced PCa who underwent single-fraction HDR-BT with EBRT at the Kanazawa University Hospital.

\section{Materials and Methods}

Patients. For this institutional review board-approved retrospective study at Kanazawa University Hospital, the medical records of 85 patients diagnosed with $\mathrm{PCa}$ who were followed for $\geq$ one year 
between April 2014 and October 2017 were included. Lesions were categorized according to the tumor-node-metastasis (TNM) classification by the International Union Against Cancer (2009). Patients were stratified into low-, intermediate-, and high-risk groups according to the D'Amico risk group classification (14), based on initial serum prostate-specific antigen (PSA) level, Gleason score (GS), and clinical tumor stage. In contrast, we defined that patients with two or more high risk factors according to D'Amico risk categories, or clinical stage T3b/T4 were reclassified into the very high-risk group. Generally, neoadjuvant hormonal therapy (NAHT) was administered to decrease prostate volume $(<50 \mathrm{ml})$ and prevent disease progression while awaiting treatment for six months. Adjuvant hormonal therapy (AHT) for two years was recommended for very high-risk patients.

High-dose-rate brachytherapy. Interstitial catheter implantation was usually carried out under spinal anaesthesia in awaking with transrectal ultrasound guidance in lithotomy position using a perineal template in an operation room. Three gold markers were inserted to mark the bilateral base and the apex of the prostate. After the catheter implantation, the patient was transferred to a computed tomography table for treatment planning. Treatment planning was conducted using Oncentra ${ }^{\circledR}$ Brachy (Nucletron). Dose constraints for prostate, rectum and urethra were V100 $>95 \%$, V75\% <1cc, V $125 \%<1 \mathrm{cc}$, respectively. Next, the patient was transferred to a treatment table in HDR unit room, and irradiation was conducted using an ${ }^{192}$ Ir microSelectron ${ }^{\circledR}$ (Nucletron) at 13 Gy in a single fraction. After the irradiation session, the needles were removed, and the patient kept the triple-lumen catheter with continuous irrigation until the next day. The following day, the urethral catheter was removed, and the patient was released. According to this singlefraction protocol, the treatment was completed in a few hours while the effect of the spinal anaesthesia remained. This surely contributed to a shorter treatment time and improved HRQOL during treatment.

External beam radiotherapy. Approximately one week after the HDR procedure, EBRT delivering $46 \mathrm{~Gy}$ in 23 fractions was initiated using intensity modulated radiotherapy for all patients with or without pelvic lymph node $(\mathrm{LN})$ metastases. Irradiation to the small pelvic cavity comprised an inner/outside ileum, obturator and sacrum anterior LN, and surely included pelvic LNs metastases. Although it has been reported that local control was enabled so that biologically effective dose (BED) was high and the biochemical freedom from failure was higher when the delivered BED was $>220$ Gy (15), BED in our protocol corresponded to 232 Gy using an $\alpha / \beta$ ratio of 1.5 Gy.

Toxicity and HRQOL evaluation. Toxicities were recorded according to the Common Terminology Criteria for Adverse Events v4.0, and adverse events less than or after three months were defined as acute or late toxicities, respectively. Responses to HRQOL questionnaires were obtained from 53 patients and the general and disease-specific HRQOL were measured using the Expanded Prostate Cancer Index Composite (EPIC), International Prostate Symptom Score (IPSS)/QOL index, International Index of Erectile Function 5 (IIEF5), and 36-Item Short Form Survey (SF-36) at pre-treatment and at one, three, six, and 12 months after brachytherapy, consistently. EPIC scoring system consisted of a 50-item questionnaire categorized into four domains (urinary, bowel, sexual, and hormonal) to quantify the PCa-specific QOL. All EPIC scores were
Table I. Patients characteristics.

\begin{tabular}{|c|c|}
\hline Number of patients & 85 \\
\hline \multicolumn{2}{|l|}{ Follow-up, months } \\
\hline Median (range) & $28.8(12.3-55.3)$ \\
\hline \multicolumn{2}{|l|}{ Age, years } \\
\hline Median (range) & $70(50-82)$ \\
\hline \multicolumn{2}{|c|}{ PSA at diagnosis, $\mathrm{ng} / \mathrm{ml}$} \\
\hline Median (range) & $17.5(1.9-557.6)$ \\
\hline$\leq 10$ & $26(30.6)$ \\
\hline$>10, \leq 20$ & $21(24.7)$ \\
\hline$>20$ & $38(44.7)$ \\
\hline \multicolumn{2}{|l|}{ Clinical stage } \\
\hline $\mathrm{T} 1 \mathrm{c}-\mathrm{T} 2 \mathrm{a}$ & $7(8.2)$ \\
\hline $\mathrm{T} 2 \mathrm{~b}$ & $11(12.9)$ \\
\hline $\mathrm{T} 2 \mathrm{c}$ & $6(7.1)$ \\
\hline T3a & $35(41.2)$ \\
\hline $\mathrm{T} 3 \mathrm{~b}$ & $19(22.4)$ \\
\hline $\mathrm{T} 4$ & $7(8.2)$ \\
\hline No & $74(87.1)$ \\
\hline N1 & $11(12.9)$ \\
\hline \multicolumn{2}{|l|}{ Gleason score } \\
\hline$\leq 6$ & $2(2.4)$ \\
\hline 7 & $26(30.6)$ \\
\hline 8 & $33(38.8)$ \\
\hline 9,10 & $23(27.1)$ \\
\hline Unknown & $1(1.2)$ \\
\hline \multicolumn{2}{|c|}{ D'Amico risk classification } \\
\hline Low & $0(0)$ \\
\hline Intermediate & $5(5.9)$ \\
\hline High & $80(94.1)$ \\
\hline \multicolumn{2}{|c|}{ Adjuvant hormonal therapy } \\
\hline Yes & $58(68.2)$ \\
\hline No & $27(31.8)$ \\
\hline \multicolumn{2}{|c|}{ Hospital length of stay, days } \\
\hline Median (range) & $10(5-20)$ \\
\hline \multicolumn{2}{|c|}{ Post-operative length of stay, days } \\
\hline Median (range) & $4(2-14)$ \\
\hline
\end{tabular}

Data presented as n (\%), unless otherwise noted.

linearly transformed to a scale of 0 (lowest) to 100 (highest). The SF-36 contained 36 items covering eight domains of HRQOL, including physical functioning, role limitations because of physical health problems, bodily pain, general health, vitality, social functioning, role limitations caused by emotional problems, and mental health. A score ranging from 0 (worst) to 100 (best) was calculated for each domain.

Statistical analyses. In this study, intervals for survivals were calculated from the first day of irradiation treatment to the event. Biochemical recurrence was determined according to the Phoenix criteria (16). Biochemical recurrence-free survival (bRFS) was estimated using the Kaplan-Meier method and differences compared with the log-rank test. To investigate the changes from baseline in different parameters (e.g., IPSS/QOL, IIEF-5, EPIC, SF-36 scores) over time, each score was tested by the Wilcoxon signed-rank test. Differences in the patients' characteristics were compared using Chisquared test, Fisher's exact test and Mann-Whitney $U$-test where appropriate. Statistical analyses were performed using GraphPad 
Table II. Patients characteristics of the high risk group.

\begin{tabular}{|c|c|c|c|}
\hline & High & Very high & $p$-Value \\
\hline Number of patients & 20 & 60 & \\
\hline \multicolumn{4}{|l|}{ Age, years } \\
\hline Median (range) & $68(53-77)$ & $70(50-82)$ & 0.527 \\
\hline \multicolumn{4}{|l|}{ PSA at diagnosis, $\mathrm{ng} / \mathrm{ml}$} \\
\hline Median (range) & $7.7(1.9-35.0)$ & $24.0(2.6-557$. & 6)<0.001 \\
\hline$\leq 10$ & $11(55.0)$ & $12(20.0)$ & \\
\hline$>10, \leq 20$ & $7(35.0)$ & $12(20.0)$ & \\
\hline$>20$ & $2(10.0)$ & $36(60.0)$ & \\
\hline Clinical stage & & & $<0.001$ \\
\hline $\mathrm{T} 1 \mathrm{c}-\mathrm{T} 2 \mathrm{a}$ & $5(25.0)$ & $0(0.0)$ & \\
\hline $\mathrm{T} 2 \mathrm{~b}$ & $6(30.0)$ & $2(3.3)$ & \\
\hline $\mathrm{T} 2 \mathrm{c}$ & $1(5.0)$ & $5(27.0)$ & \\
\hline T3a & $8(40.0)$ & $27(45.0)$ & \\
\hline $\mathrm{T} 3 \mathrm{~b}$ & $0(0.0)$ & $19(31.7)$ & \\
\hline $\mathrm{T} 4$ & $0(0.0)$ & $7(11.7)$ & \\
\hline No & $20(100)$ & $49(81.7)$ & 0.057 \\
\hline N1 & $0(0.0)$ & $11(18.3)$ & \\
\hline Gleason score & & & 0.003 \\
\hline$\leq 6$ & $1(5.0)$ & $0(0.0)$ & \\
\hline 7 & $10(50.0)$ & $12(20.0)$ & \\
\hline 8 & $9(45.0)$ & $24(40.0)$ & \\
\hline 9,10 & $0(0.0)$ & $23(38.3)$ & \\
\hline Unknown & $0(0.0)$ & $1(1.7)$ & \\
\hline Adjuvant hormonal therapy & & & $<0.001$ \\
\hline Yes & $6(30.0)$ & $52(86.7)$ & \\
\hline No & $14(70.0)$ & $8(13.3)$ & \\
\hline
\end{tabular}

Data presented as n (\%), unless otherwise noted.

Prism version 6.04 (GraphPad Software Inc., San Diego, CA, USA), and statistical significance was defined as a $p$-value of $<0.05$.

\section{Results}

Patient characteristics are shown in Table I. The median follow-up duration was 28.8 months (range=12.3-55.3 months), and the patients' median age was 70 years (range $=50-82$ years). Of the 85 patients included in this study, seven $(8.2 \%)$ were in clinical stage T1c-T2a, 11 $(12.9 \%)$ in $\mathrm{T} 2 \mathrm{~b}, \operatorname{six}(7.1 \%)$ in $\mathrm{T} 2 \mathrm{c}, 35(41.2 \%)$ in $\mathrm{T} 3 \mathrm{a}, 19$ $(22.4 \%)$ in $\mathrm{T} 3 \mathrm{~b}$, and seven $(8.2 \%)$ in $\mathrm{T} 4$. Eleven patients (12.9\%) had regional LN metastases. The median initial PSA level was $17.5 \mathrm{ng} / \mathrm{ml}($ range $=1.9-557.6 \mathrm{ng} / \mathrm{ml})$, and the GS was $\leq 6(2,2.4 \%),=7(26,30.6 \%),=8(33,38.8 \%), \geq 9(23$, $27.1 \%)$, and unknown $(1,1.2 \%)$, respectively. According to the D'Amico risk classification criteria, five patients $(5.9 \%)$ were at intermediate-risk, and $80(94.1 \%)$ were at high-risk. All patients underwent NAHT for a median of six months, and 58 patients $(68.2 \%)$ received AHT for two years. The median hospitalization and post-operative length of stay of patients were 10 days (range $=5-20$ days) and 4 days (range=2-14 days), respectively. In contrast, according to our modified D'Amico risk classification criteria for the high-
Table III. Acute and late toxicities according to the Common Terminology Criteria for Adverse Events v4.0.

\begin{tabular}{lccc}
\hline & $\begin{array}{c}\text { Grade 1 } \\
\mathrm{N}(\%)\end{array}$ & $\begin{array}{c}\text { Grade 2 } \\
\mathrm{N}(\%)\end{array}$ & $\begin{array}{c}\text { Grade 3 } \\
\mathrm{N}(\%)\end{array}$ \\
\hline $\begin{array}{l}\text { Acute toxicities ( 3 months) } \\
\text { Pollakisuria }\end{array}$ & $7(8.2)$ & $33(38.8)$ & $0(0)$ \\
Urgency/Incontinence & $10(11.8)$ & $1(1.2)$ & $0(0)$ \\
Urinary retention & $3(3.5)$ & $4(4.7)$ & $0(0)$ \\
Pain on urination & $6(7.1)$ & $0(0)$ & $0(0)$ \\
Hematuria & $1(1.2)$ & $4(4.7)$ & $1(1.2)$ \\
Epididymitis & $0(0)$ & $1(1.2)$ & $0(0)$ \\
Diarrhea & $18(21.2)$ & $3(3.5)$ & $0(0)$ \\
& & & \\
Late toxicities (3 months ) & & & \\
Radiation-induced urethritis & $2(2.4)$ & $0(0)$ & $0(0)$ \\
Rectal hemorrhage & $1(1.2)$ & $1(1.2)$ & $0(0)$ \\
\hline
\end{tabular}

risk group, significant differences between the high- and very high-risk group regarding the initial PSA level, clinical T stage, GS and the treatment with AHT were observed as shown in Table II.

In the present study, only three patients had biochemical recurrence during the observation period and 3-year bRFS rate was $94.4 \%$, as shown in Figure 1. Moreover, the 3-year bRFS rates for intermediate-, high-, and very high-risk patients (Figure 2 ) were $100 \%, 92.9 \%$, and $94.8 \%$, respectively ( $p=0.857$ ). The characteristics of three patients who developed biochemical recurrence were Stage: T3aNOM0 in TNM classification, initial PSA level: $60.0 \mathrm{ng} / \mathrm{ml}$, GS: 7, and Stage: T3aN1M1b, initial PSA level: $112.0 \mathrm{ng} / \mathrm{ml}$, GS: 8 , and Stage: T3aN0M0, initial PSA level: $12.5 \mathrm{ng} / \mathrm{ml}$, GS: 7, respectively. These two patients were classified into the very high-risk group.

Figure 3 shows the longitudinal changes in the mean IPSS, QOL index, and IIEF-5 scores. The IPSS and QOL scores showed a similar tendency to drop significantly at one month after treatment as compared with the baseline $(p<0.05)$ and then to recover gradually to the pre-treatment state. The IIEF-5 scores showed a significant decrease until six months after treatment. It was speculated that a high IIEF-5 score in several patients before treatment was not recovered by treatment, including NAHT and AHT. In contrast, there were no significant differences in IPSS, QOL index and IIEF-5 scores between the high- and very highrisk group one year after treatment (data not shown).

Figure 4 shows the longitudinal changes in the mean EPIC scores for the urinary, bowel, sexual, and hormonal domains and subscales. EPIC scores for the urinary domain dropped significantly at one and three months after treatment as compared with baseline, respectively $(p<0.05)$, and then recovered gradually to the pre-treatment state six months after treatment. The bowel domain also dropped significantly 


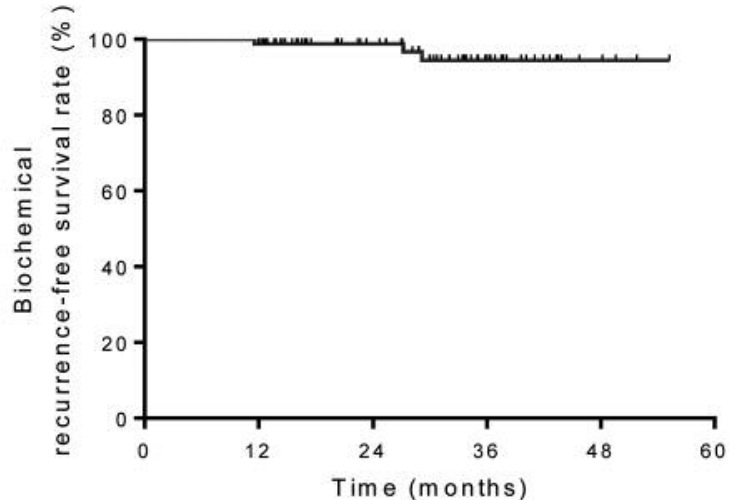

Numbers at risk

$\begin{array}{lllll}85 & 84 & 53 & 24 & 6\end{array}$

Figure 1. Biochemical recurrence-free survival rates for all patients.

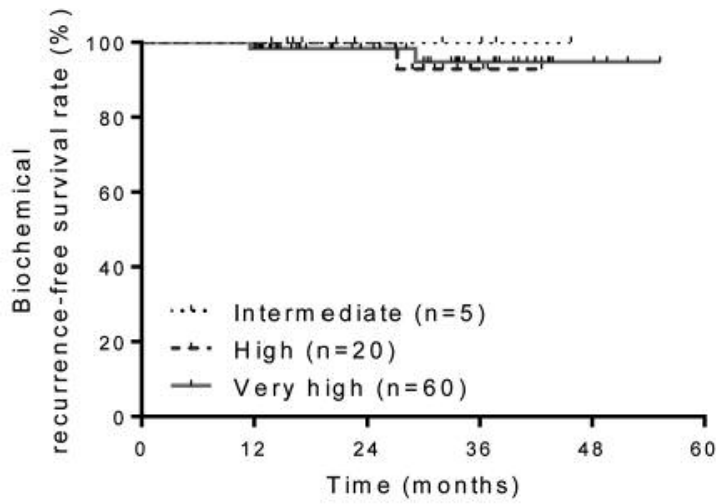

Numbers at risk

$\begin{array}{cccccc}\text { Intermediate } & 5 & 5 & 5 & 4 & 1 \\ \text { High } & 20 & 20 & 15 & 4 & 1 \\ \text { Very high } & 60 & 59 & 35 & 18 & 6\end{array}$

Figure 2. Biochemical recurrence-free survival rates stratified by the modified-risk group.

until six months after treatment as compared with baseline $(p<0.05)$. In contrast, there was a temporary deterioration at one month after treatment in the sexual function, but the hormonal domain improved significantly after treatment as compared with the baseline $(p<0.05)$, because AHT was interrupted in about $30 \%$ of cases, as shown in Table I. Although there was not any difference in urinary and bowel function between high- and very high-risk group one year after treatment, sexual and hormonal function in the very high-risk group dropped significantly six and 12 months after treatment, respectively (data not shown), because most of the very high-risk patients received AHT, as shown in Table II.
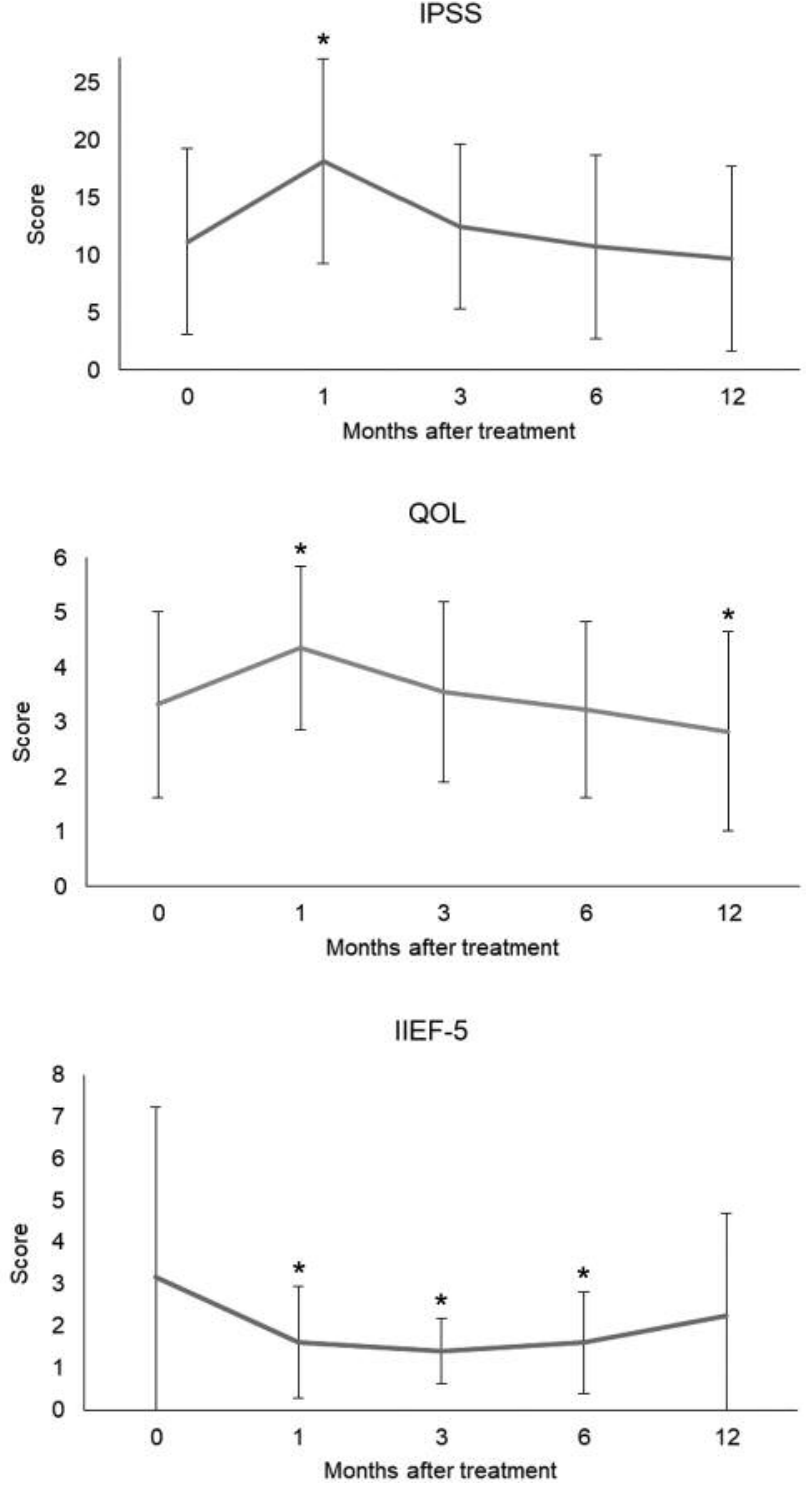

Figure 3. Longitudinal changes in International Prostate Symptom Score, QOL, and International Index of Erectile Function $5(* p<0.05$, vs. pre-treatment).

Figure 5 shows the longitudinal changes in mean SF-36 scores. "Role physical (RP)," "General health," "Vitality," "Social functioning (SF)," and "Role emotional (RE)" showed temporarily significant exacerbation at one month after treatment. A reduction in quality of life during the duration of external irradiation treatment may cause exacerbation, because patients rarely complained after HDR-BT. In contrast, RP in the very high-risk group showed significant exacerbation until about 12 months after treatment compared with the high-risk group, similar to SF and RE (data not shown). 


\section{(a) Urinary domain}
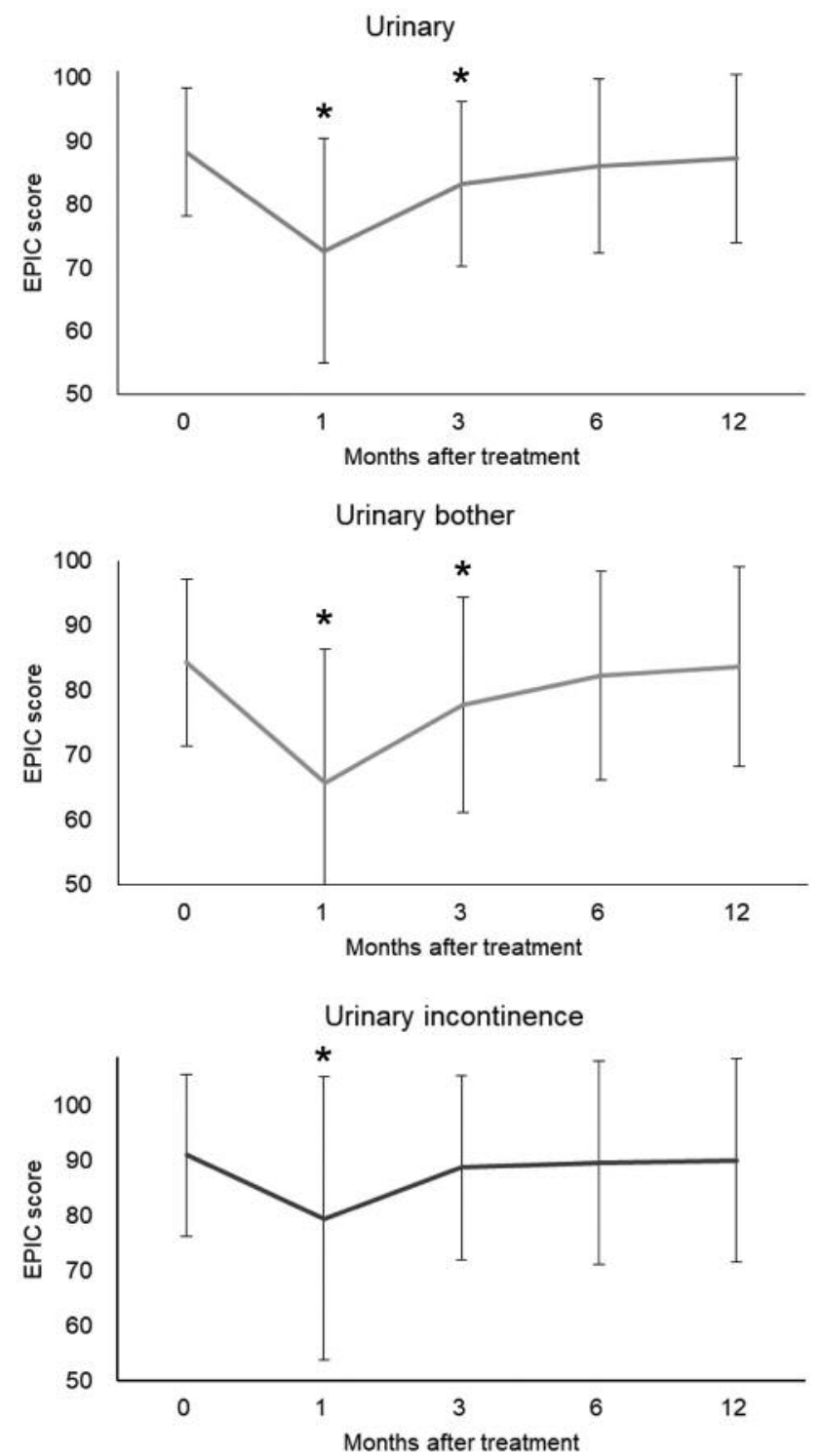

Toxicities are described in Table III. The most frequent acute genitourinary complications were pollakisuria, followed by urgency/incontinence, both below grade 2 toxicities. Although urethral retention was found in seven patients $(8.2 \%)$ in the acute phase, urinary stricture causing urethral retention in the late phase, which was often observed as an adverse effect of conventional fractionated HDR-BT, was not observed during the follow-up period. Acute gastrointestinal complication was mainly found as grade 1 diarrhea, and only two patients $(2.4 \%)$ experienced rectal hemorrhage and radiation-induced urethritis in the late phase, respectively. Although most toxicities were below grade 2,
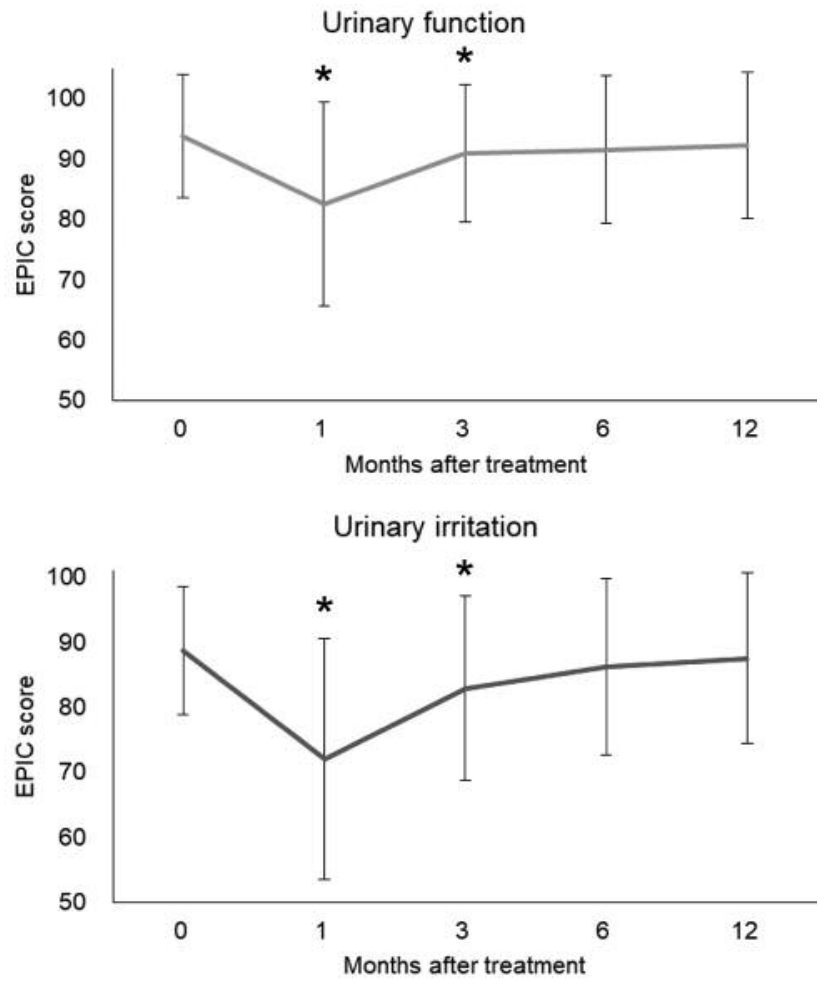

Figure 4. Continued

one patient $(1.2 \%)$ experienced grade 3 hematuria. In contrast, there were no significant differences in the frequency of adverse events between the high- and very high-risk group (data not shown).

\section{Discussion}

In recent years, many institutions have reported treatment outcomes of HDR-BT for patients with $\mathrm{PCa}$ and proven excellent bRFS rates for long-term outcome data. According to our previous study, the 5-year bRFS rates for patients in the low-, intermediate-, and high-risk groups were $100 \%$, 
(b) Bowel domain
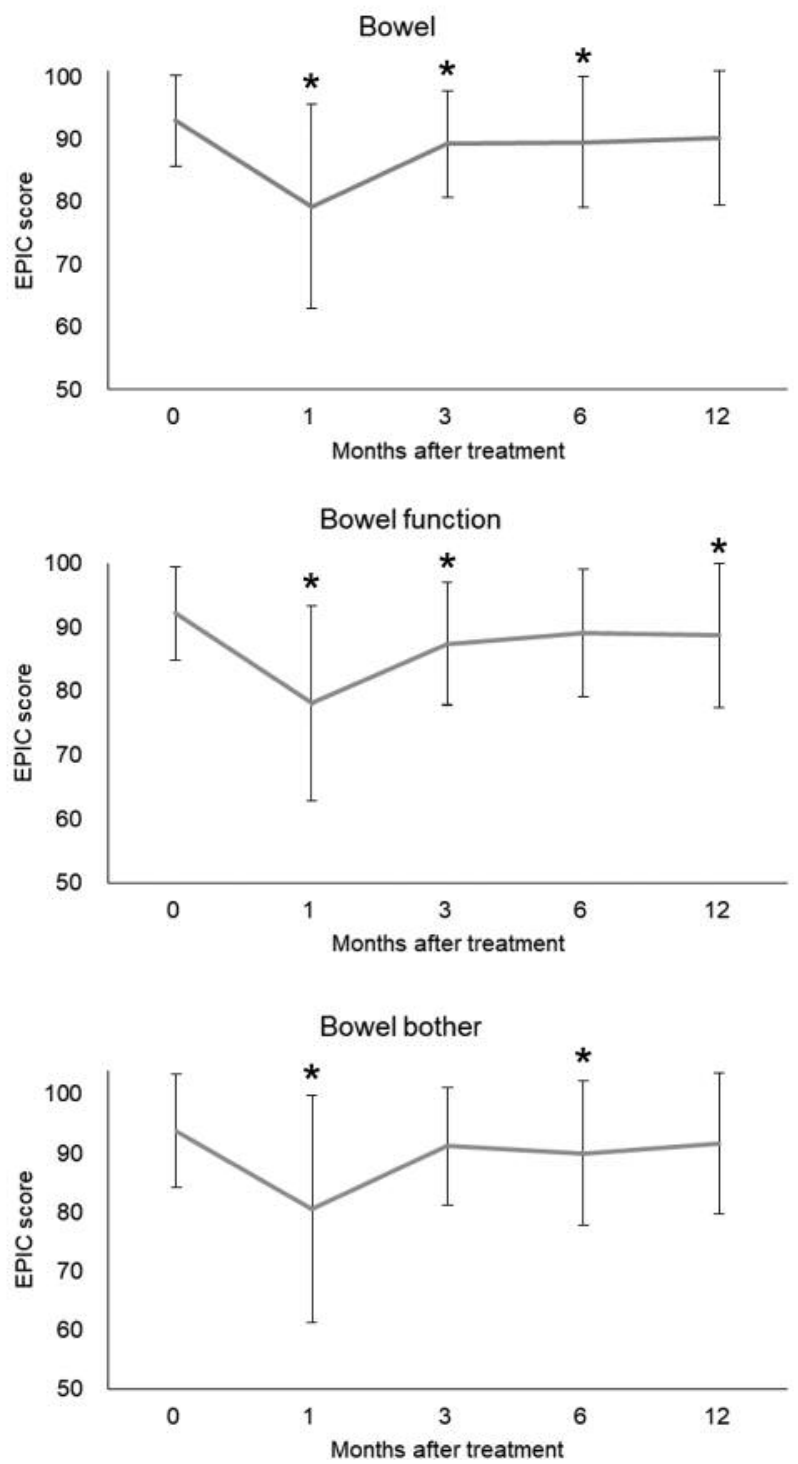

$95.6 \%$, and $90.7 \%$, respectively. This is a promising result, like past reports (5). Although there is no consensus on the optimal dose and time/fractionation schedule for HDR-BT, recent trends are moving toward a smaller number of fractions and shorter treatment times, because single-fraction HDR-BT is a very attractive alternative $(6-10,13)$. Moreover, the need to measure value in healthcare has become increasingly pressing, and quality of life issues have gained prominence in treatment decision-making (17).

To date, in Japan, there is no study on single-fraction HDR-BT to evaluate its efficacy, safety, tolerance, and

\section{(c) Sexual domain}
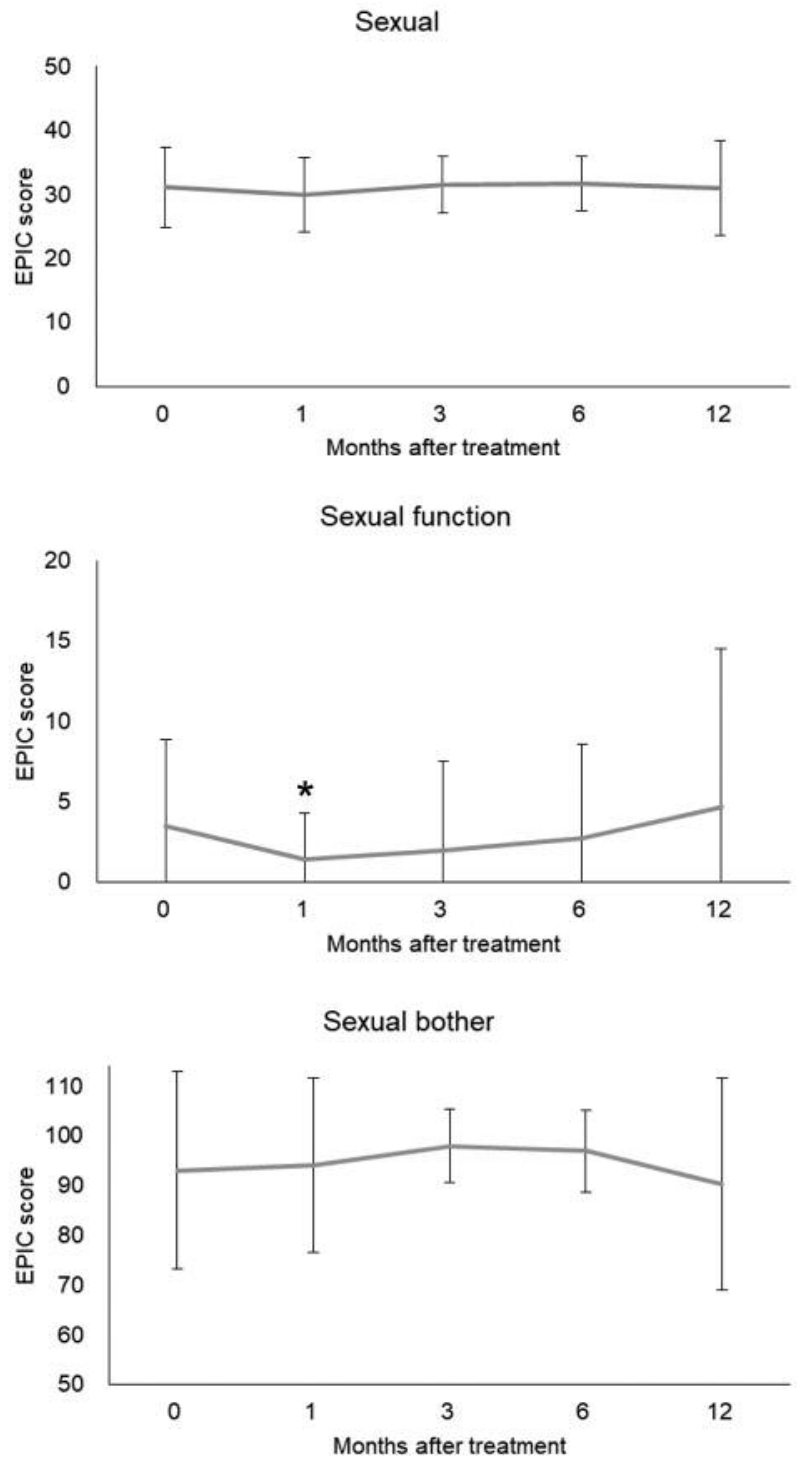

Figure 4. Continued

impact on HRQOL. In the present study, although the observation period was short and there were many high- and very high-risk patients, a lower rate of biochemical recurrence was seen, in comparison to previous studies (13). The notable points can be given as reasons to insert the applicator into a seminal vesicle and place it in the irradiation fields when cancer has invaded a seminal vesicle. Moreover, hormonal therapies are used together effectively, contributing to the improvement of biochemical control. Although acute adverse events are mainly genitourinary toxicities, such as pollakisuria, urgency/incontinence, and 
(d) Hormonal domain
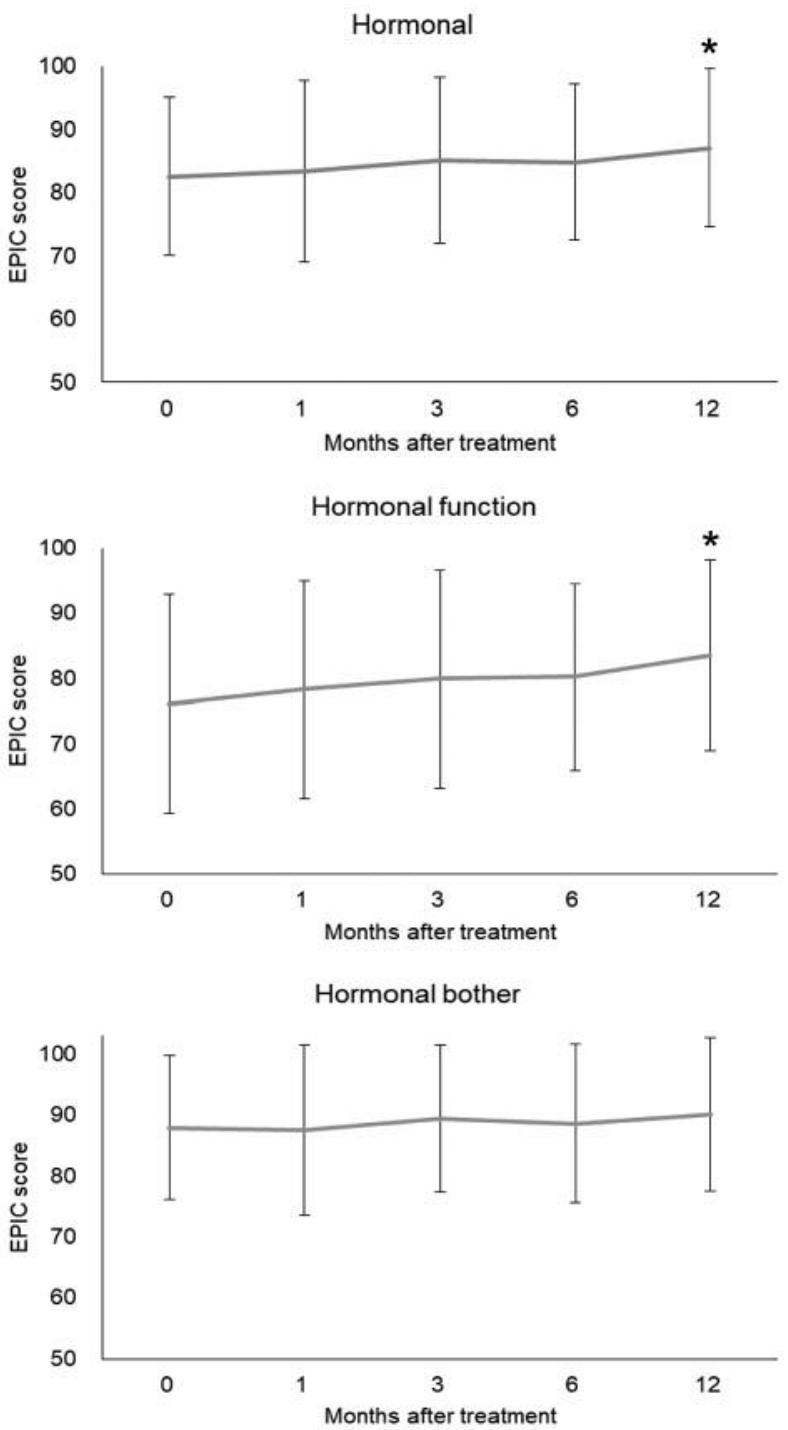

Figure 4. Longitudinal changes in the Expanded Prostate Cancer Index Composite scores. EPIC domains: (a) urinary; (b) bowel; (c) sexual; and $(d)$ hormonal $(* p<0.05, v s$. pre-treatment $)$.

low-grade diarrhea, almost all of them are easily managed by temporary medication. These results are comparable to previously reported data, in which acute grade 1,2 , and 3 urinary toxicity occurred in $35 \%, 62 \%$, and $1.6 \%$ of patients, respectively, and acute grade 1,2 , and 3 bowel toxicity occurred in $59 \%, 7 \%$, and $0 \%$ of patients, respectively, after HDR-BT in combination with EBRT (18). Our study included a cohort of patients with high- and very high-risk $\mathrm{PCa}$; therefore, receiving a combined modality treatment of hormonal therapy and EBRT may contribute to acute genitourinary and gastrointestinal toxicities. In contrast, late adverse events were only seen in two cases of low-grade urethritis and rectal hemorrhage. Several studies have reported cases of urethral stricture following conventional HDR-BT with EBRT, with a urethral stricture rate of 2-10\% $(2,19,20)$. Our previous study $(19 \mathrm{~Gy} / 2$ fractions of HDRBT until March 2014) showed a similar rate of urethral stricture $(8.8 \%)$, and most urethral stricture occurred within two years of HDR-BT (5). In contrast, urethral stricture was not observed in this study. Although the migration of applicators during two or three fractions of HDR-BT may cause excess irradiation to a distal portion of the urethral sphincter, a single fraction of HDR-BT generally may reduce the risk of migration of applicators.

Although few reports focus on HRQOL after single-fraction HDR-BT, our results are comparable with previous studies. Gomez et al. (21) reported a statistically significant decline in EPIC urinary urgency/obstructive domain at the third month and recovery to baseline by the sixth month. Mean EPIC urinary incontinence, and bowel, sexual, and hormonal domains were not changed significantly post-treatment with 19 Gy single-fraction HDR-BT. Shahid et al. (22) published a phase II clinical trial of 15 Gy HDR boost and EBRT to a dose of $37.5 \mathrm{~Gy}$ in 15 fractions for patients with intermediate-risk PCa followed for a long-term, like our HDR-BT protocol. They observed a significant change in the median EPIC scores from baseline to year five in the urinary, bowel, and sexual domains, but the hormonal domain remained unchanged. Quality of life in the present study showed that mean EPIC scores for the urinary and bowel domains drop significantly at three and six months after treatment compared with the baseline $(p<0.05)$, and then recover gradually to the pre-treatment state. In contrast, there was a temporary deterioration in the sexual function at one month after treatment, but the hormonal domain improved significantly at 12 months after treatment as compared with the baseline $(p<0.05)$. Interruption of hormonal therapy after irradiation may have contributed to the improvement of EPIC hormonal scores.

The present study is limited by the fact that it is a retrospective study, and due to the short follow-up of the cohort, the data are not yet sufficiently mature to report on efficacy outcomes. Further, it is necessary to consider that this study adopts an $\alpha / \beta$ ratio of 1.5 Gy unlike the fact that Dr. Stone reported BED was an important parameter of biochemical recurrence using an $\alpha / \beta$ ratio of 2 Gy (15). Another limitation of this study is that we were not able to evaluate the differences potentially contributing to genitourinary and gastrointestinal toxicities, as well as changes in HRQOL between the radiation dose from the single-fraction interstitial irradiation and the external beam component. Moreover, the pre-treatment HRQOL questionnaire was obtained just before HDR-BT treatment, so that the effect of NAHT cannot be ignored. However, single-fraction HDR-BT 
Physical functioning

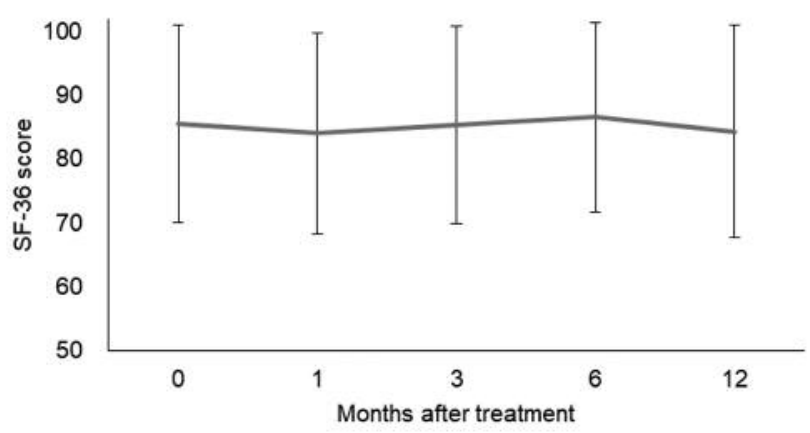

Bodily pain

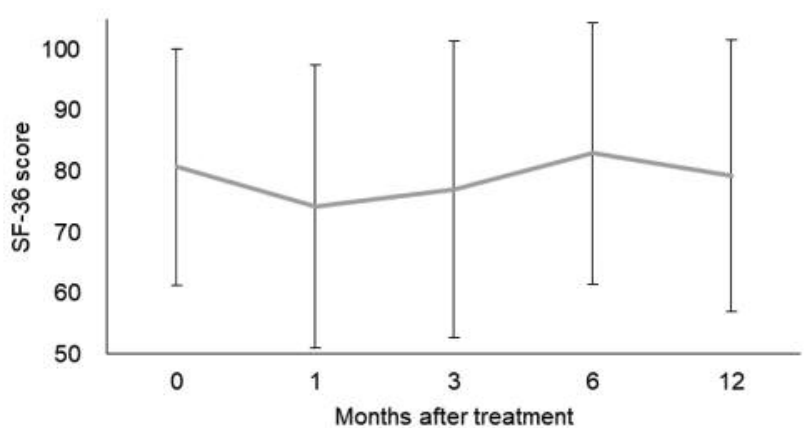

Vitality

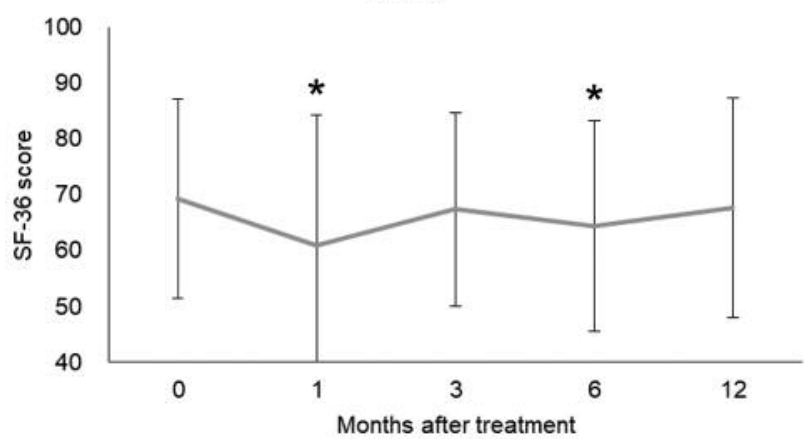

Role emotional

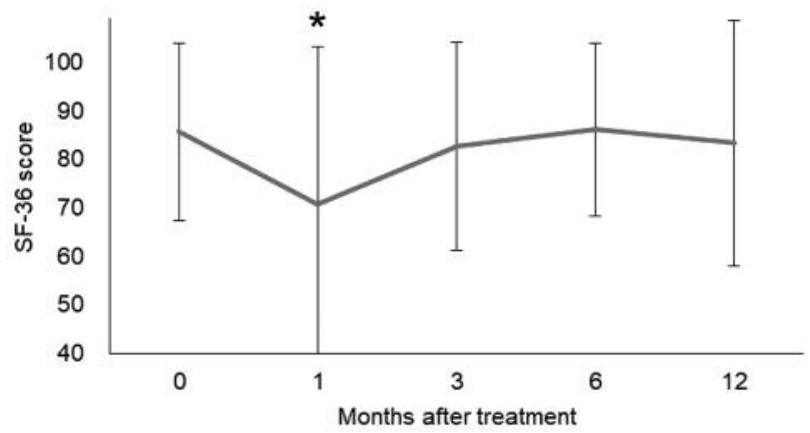

Role physical

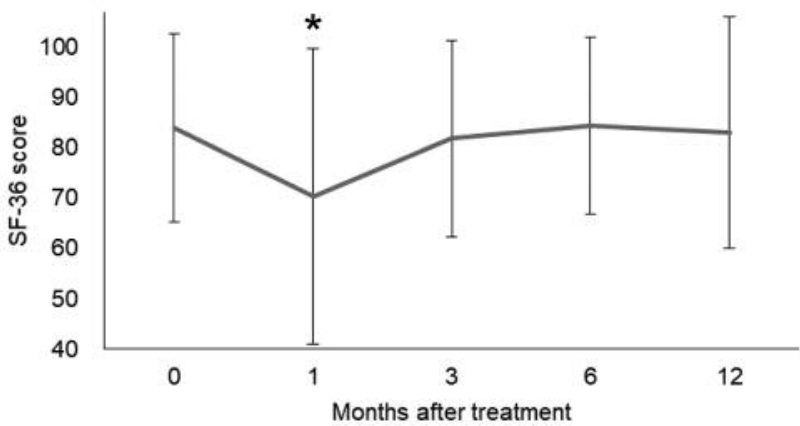

General health

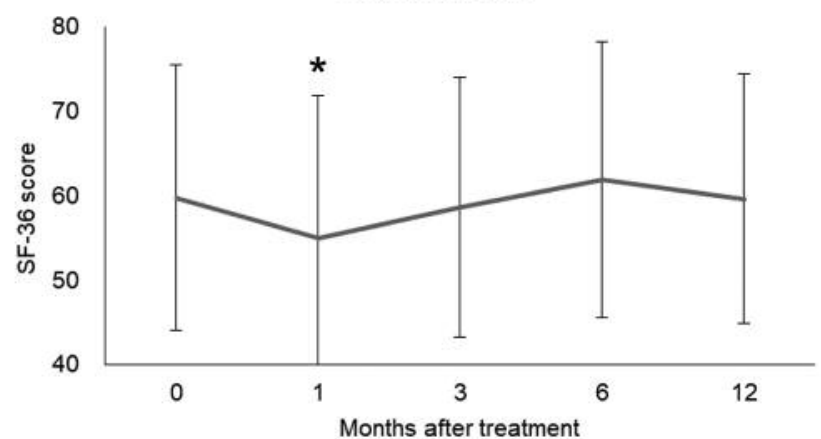

Social functioning

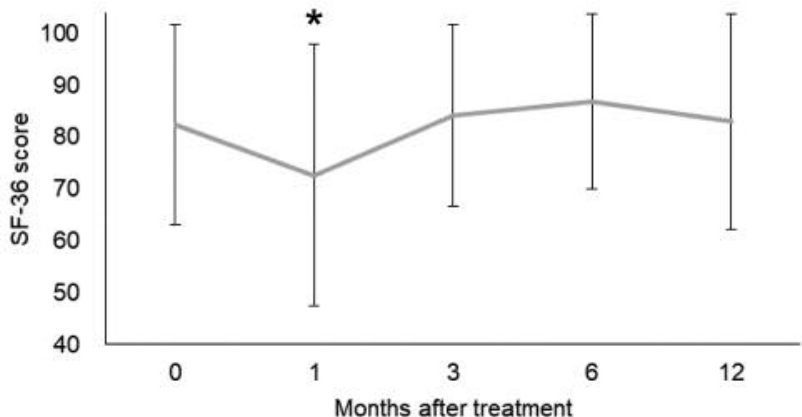

Mental health

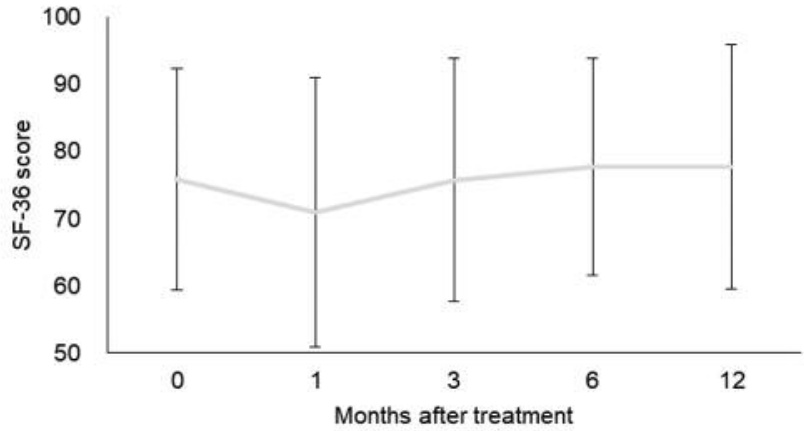

Figure 5. Longitudinal changes in the 36-Item Short Form Survey $(* p<0.05$, vs. pre-treatment). 
combined with EBRT improved the biochemical control in localized and locally advanced PCa, and it can be delivered with tolerant toxicities, temporary detrimental impact on HRQOL, and high satisfaction of both patients and clinicians through shorter treatment times. Interestingly, it is notable that urethral stricture, which is a well-known late toxicity-related problem was not observed.

In conclusion, the clinical outcomes of patients with localized and locally advanced PCa who underwent singlefraction HDR-BT combined with EBRT are reported. Although excellent results were obtained regarding biological control, safety, impact on HRQOL, and high satisfaction of both patients and clinicians because of shorter treatment times, a longer follow-up period is required to assess the effect of this single-fraction dose protocol on long-term biological control.

\section{Conflicts of Interest}

No potential conflict of interest relevant to this article was reported.

\section{References}

1 Ishiyama H, Kamitani N, Kawamura H, Kato S, Aoki M, Kariya S, Matsumura T, Kaidu M, Yoshida K, Hashimoto Y, Noda Y, Lim KHC, Kawase T, Takahashi T, Inaba K, Kumano M, Yoshikawa N, Yoshioka Y, Nakamura K, Hiratsuka J, Itami $\mathrm{J}$ and Hayakawa K: Nationwide multi-institutional retrospective analysis of high-dose-rate brachytherapy combined with external beam radiotherapy for localized prostate cancer: An asian prostate hdr-bt consortium. Brachytherapy 16(3): 503-510, 2017.

2 Hoskin PJ, Rojas AM, Bownes PJ, Lowe GJ, Ostler PJ and Bryant L: Randomised trial of external beam radiotherapy alone or combined with high-dose-rate brachytherapy boost for localised prostate cancer. Radiother Oncol 103(2): 217-222, 2012.

3 Strouthos I, Chatzikonstantinou G, Zamboglou N, Milickovic N, Papaioannou S, Bon D, Zamboglou C, Rodel C, Baltas D and Tselis N: Combined high dose rate brachytherapy and external beam radiotherapy for clinically localised prostate cancer. Radiother Oncol 128(2): 301-307, 2018.

4 Kotecha R, Yamada Y, Pei X, Kollmeier MA, Cox B, Cohen GN, Zaider M and Zelefsky MJ: Clinical outcomes of high-doserate brachytherapy and external beam radiotherapy in the management of clinically localized prostate cancer. Brachytherapy 12(1): 44-49, 2013.

5 Makino T, Mizokami A and Namiki M: Clinical outcomes of patients with localized and locally advanced prostate cancer undergoing high-dose-rate brachytherapy with external-beam radiotherapy at our institute. Anticancer Res 35(3): 1723-1728, 2015.

6 Krauss DJ, Ye H, Martinez AA, Mitchell B, Sebastian E, Limbacher A and Gustafson GS: Favorable preliminary outcomes for men with low- and intermediate-risk prostate cancer treated with 19-gy single-fraction high-dose-rate brachytherapy. Int $\mathbf{J}$ Radiat Oncol Biol Phys 97(1): 98-106, 2017.
7 Morton G, Chung HT, McGuffin M, Helou J, D'Alimonte L, Ravi A, Cheung P, Szumacher E, Liu S, Al-Hanaqta M, Zhang L, Mamedov A and Loblaw A: Prostate high dose-rate brachytherapy as monotherapy for low and intermediate risk prostate cancer: Early toxicity and quality-of life results from a randomized phase ii clinical trial of one fraction of $19 \mathrm{gy}$ or two fractions of 13.5gy. Radiother Oncol 122(1): 87-92, 2017.

8 Hoskin P, Rojas A, Ostler P, Hughes R, Alonzi R and Lowe G: Single-dose high-dose-rate brachytherapy compared to two and three fractions for locally advanced prostate cancer. Radiother Oncol 124(1): 56-60, 2017.

9 Prada PJ, Cardenal J, Blanco AG, Anchuelo J, Ferri M, Fernandez G, Arrojo E, Vazquez A, Pacheco M and Fernandez $\mathrm{J}$ : High-dose-rate interstitial brachytherapy as monotherapy in one fraction for the treatment of favorable stage prostate cancer: Toxicity and long-term biochemical results. Radiother Oncol 119(3): 411-416, 2016.

10 Falk AT, Demontoy S, Chamorey E, Chand ME, Gautier M, Azria D, Zaki S, Chevallier D, Cham Kee DL and Hannoun-Levi JM: High-dose-rate brachytherapy boost for prostate cancer: Comparison of three different fractionation schemes. Brachytherapy 16(5): 993-999, 2017.

11 Hijazi H, Chevallier D, Gal J, Chand ME, Gautier M and Hannoun-Levi JM: Prostate cancer boost using high-dose-rate brachytherapy: Early toxicity analysis of 3 different fractionation schemes. J Contemp Brachytherapy 5(4): 203-209, 2013.

12 Lauche O, Delouya G, Taussky D, Menard C, Beliveau-Nadeau D, Hervieux Y, Larouche R and Barkati M: Single-fraction highdose-rate brachytherapy using real-time transrectal ultrasound based planning in combination with external beam radiotherapy for prostate cancer: Dosimetrics and early clinical results. J Contemp Brachytherapy 8(2): 104-109, 2016.

13 Boladeras A, Santorsa L, Gutierrez C, Martinez E, Pera J, Pino F, Suarez JF, Ferrer F, Diaz A, Polo A and Guedea F: External beam radiotherapy plus single-fraction high dose rate brachytherapy in the treatment of locally advanced prostate cancer. Radiother Oncol 112(2): 227-232, 2014.

14 D'Amico AV, Whittington R, Malkowicz SB, Schultz D, Blank K, Broderick GA, Tomaszewski JE, Renshaw AA, Kaplan I, Beard CJ and Wein A: Biochemical outcome after radical prostatectomy, external beam radiation therapy, or interstitial radiation therapy for clinically localized prostate cancer. JAMA 280(11): 969-974, 1998.

15 Stone NN, Potters L, Davis BJ, Ciezki JP, Zelefsky MJ, Roach M, Shinohara K, Fearn PA, Kattan MW and Stock RG: Multicenter analysis of effect of high biologic effective dose on biochemical failure and survival outcomes in patients with gleason score 7-10 prostate cancer treated with permanent prostate brachytherapy. Int J Radiat Oncol Biol Phys 73(2): 341346, 2009.

16 Roach M, 3rd, Hanks G, Thames H Jr., Schellhammer P, Shipley WU, Sokol GH and Sandler H: Defining biochemical failure following radiotherapy with or without hormonal therapy in men with clinically localized prostate cancer: Recommendations of the rtog-astro phoenix consensus conference. Int J Radiat Oncol Biol Phys 65(4): 965-974, 2006.

17 Porter ME: What is value in health care? N Engl J Med 363(26): 2477-2481, 2010.

18 Morton GC, Loblaw DA, Chung H, Tsang G, Sankreacha R, Deabreu A, Zhang L, Mamedov A, Cheung P, Batchelar D, 
Danjoux C and Szumacher E: Health-related quality of life after single-fraction high-dose-rate brachytherapy and hypofractionated external beam radiotherapy for prostate cancer. Int J Radiat Oncol Biol Phys 80(5): 1299-1305, 2011.

19 Ishiyama H, Satoh T, Kitano M, Tabata K, Komori S, Ikeda M, Soda I, Kurosaka S, Sekiguchi A, Kimura M, Kawakami S, Iwamura $\mathrm{M}$ and Hayakawa K: High-dose-rate brachytherapy and hypofractionated external beam radiotherapy combined with long-term hormonal therapy for high-risk and very high-risk prostate cancer: Outcomes after 5-year follow-up. J Radiat Res 55(3): 509-517, 2014.

20 Sullivan L, Williams SG, Tai KH, Foroudi F, Cleeve L and Duchesne GM: Urethral stricture following high dose rate brachytherapy for prostate cancer. Radiother Oncol 91(2): 232236,2009
21 Gomez-Iturriaga A, Casquero F, Pijoan JI, Minguez P, Espinosa JM, Irasarri A, Bueso A, Cacicedo J, Buchser D and Bilbao P: Health-related-quality-of-life and toxicity after single fraction 19gy high-dose-rate prostate brachytherapy: Phase ii trial. Radiother Oncol 126(2): 278-282, 2018.

22 Shahid N, Loblaw A, Chung HT, Cheung P, Szumacher E, Danjoux C, Sankreacha R, Zhang L, Deabreu A, Mamedov A and Morton G: Long-term toxicity and health-related quality of life after single-fraction high dose rate brachytherapy boost and hypofractionated external beam radiotherapy for intermediate-risk prostate cancer. Clin Oncol (R Coll Radiol) 29(7): 412-420, 2017.

Received November 26, 2018

Revised December 3, 2018

Accepted December 4, 2018 